\title{
BMJ Open Comparative safety and effectiveness of long-acting inhaled agents for treating chronic obstructive pulmonary disease: a systematic review and network meta-analysis
}

Andrea C Tricco, ${ }^{1,2}$ Lisa Strifler, ${ }^{1}$ Areti-Angeliki Veroniki, ${ }^{1}$ Fatemeh Yazdi, ${ }^{3}$ Paul A Khan, ${ }^{1}$ Alistair Scott, ${ }^{1}$ Carmen Ng, ${ }^{1}$ Jesmin Antony, ${ }^{1}$ Kelly Mrklas, ${ }^{1,4}$ Jennifer D'Souza, ${ }^{1}$ Roberta Cardoso, ${ }^{1}$ Sharon E Straus ${ }^{1,5}$

To cite: Tricco AC, Strifler $L$, Veroniki A-A, et al. Comparative safety and effectiveness of long-acting inhaled agents for treating chronic obstructive pulmonary disease: a systematic review and network meta-analysis. BMJ Open 2015;5:e009183. doi:10.1136/bmjopen-2015009183

- Prepublication history and additional material is available. To view please visit the journal (http://dx.doi.org/ 10.1136/bmjopen-2015009183)

Received 23 June 2015 Revised 16 September 2015 Accepted 6 October 2015

CrossMark

For numbered affiliations see end of article.

Correspondence to Dr Sharon E Straus; sharon.straus@utoronto.ca

\section{ABSTRACT}

Objective: To compare the safety and effectiveness of long-acting $\beta$-antagonists (LABA), long-acting antimuscarinic agents (LAMA) and inhaled corticosteroids (ICS) for managing chronic obstructive pulmonary disease (COPD).

Setting: Systematic review and network meta-analysis (NMA).

Participants: 208 randomised clinical trials (RCTs) including 134692 adults with COPD.

Interventions: LABA, LAMA and/or ICS, alone or in combination, versus each other or placebo.

Primary and secondary outcomes: The proportion of patients with moderate-to-severe exacerbations. The number of patients experiencing mortality, pneumonia, serious arrhythmia and cardiovascular-related mortality (CVM) were secondary outcomes.

Results: NMA was conducted including 20 RCTs for moderate-to-severe exacerbations for 26141 patients with an exacerbation in the past year. 32 treatments were effective versus placebo including: tiotropium, budesonide/formoterol, salmeterol, indacaterol, fluticasone/salmeterol, indacaterol/glycopyrronium, tiotropium/fluticasone/salmeterol and tiotropium/ budesonide/formoterol. Tiotropium/budesonide/ formoterol was most effective $(99.2 \%$ probability of being the most effective according to the Surface Under the Cumulative RAnking (SUCRA) curve). NMA was conducted on mortality (88 RCTs, 97526 patients); fluticasone/salmeterol was more effective in reducing mortality than placebo, formoterol and fluticasone alone, and was the most effective (SUCRA $=71 \%$ ). NMA was conducted on CVM (37 RCTs, 55156 patients) and the following were safest: salmeterol versus each OF placebo, tiotropium and tiotropium (Soft Mist Inhaler (SMR)); fluticasone versus tiotropium (SMR); and salmeterol/fluticasone versus tiotropium and tiotropium (SMR). Triamcinolone acetonide was the most harmful (SUCRA $=81 \%$ ). NMA was conducted on pneumonia occurrence (54 RCTs, 61551 patients). 24 treatments were more harmful, including 2 that increased risk of
Strengths and limitations of this study

- We included $>200$ randomised trials and this is one of the most comprehensive systematic reviews in this area.

- We follow the methodologically rigorous guidelines put forth by the Cochrane Collaboration.

- We conducted a network meta-analysis in accordance with guidance put forth by the International Society For Pharmacoeconomics and Outcomes Research.

- Many of the included randomised trials were at a high risk of bias for many of the Cochrane risk-of-bias criteria.

- We were unable to explore other important effect modifiers, such as duration of treatment administration, as this was inconsistently reported across the included randomised trials.

pneumonia versus placebo; fluticasone and fluticasone/ salmeterol. The most harmful agent was fluticasone/ salmeterol (SUCRA $=89 \%)$. NMA was conducted for arrhythmia; no statistically significant differences between agents were identified.

Conclusions: Many inhaled agents are available for COPD, some are safer and more effective than others. Our results can be used by patients and physicians to tailor administration of these agents.

Protocol registration number: PROSPERO \# CRD42013006725.

\section{BACKGROUND}

Evidence from previous systematic reviews and network meta-analyses suggests that inhaled therapy with inhaled corticosteroids (ICS), long-acting $\beta$-agonists (LABA), and long-acting muscarinic antagonists (LAMA) for patients with chronic obstructive 
pulmonary disease (COPD) is promising. ${ }^{1-9}$ However, to date, it is not clear which combinations of inhaled therapies are the safest and most effective for these patients for all relevant outcomes. In order to examine this issue further, we conducted a systematic review and network meta-analysis. This work is part of a Drug Class Review conducted by the Ontario Drug Policy Research Network (ODPRN) that was funded by the Ontario Ministry of Health and Long-Term Care Health System Research Fund. Our research question was 'What is the comparative safety and effectiveness of long-acting inhaled agents (ICS, LABA, LAMA), alone or in any combination, for patients with COPD?'

\section{METHODS}

\section{Protocol}

Our systematic review protocol was drafted using the Preferred Reporting Items for Systematic reviews and Meta-analyses for Protocols (PRISMA-P) guidance. ${ }^{10}$ The protocol was revised based on feedback from various stakeholders, including policymakers from the Ontario Public Drug Programs, industry stakeholders, patients, researchers within the ODPRN and healthcare professionals. The final protocol was registered with the PROSPERO registry (CRD42013006725) and posted on the ODPRN website. ${ }^{11}$ Since our full methods have been posted online, they are summarised briefly here.

\section{Eligibility criteria}

Parallel-group randomised clinical trials (RCTs) including adults with COPD administered long-acting inhaled agent in any combination compared with each other or placebo were eligible for inclusion. Concomitant COPD medications were included if both groups received the same interventions (eg, rescue medication with a shortacting $\beta$-agonist). A list of included agents can be found in online supplementary appendix 1. A list of the excluded medications can be found in online supplementary appendix 2.

The primary outcome of interest was the proportion of patients with moderate-to-severe exacerbations (ie, worsening of COPD symptoms that may require hospitalisation, emergency department visits, treatment with oral steroids and/or antibiotics, use of rescue medication, or unscheduled visits to a walk-in clinic or to a healthcare provider). Secondary outcomes included the number of patients experiencing mortality, pneumonia, serious arrhythmia and cardiovascular-related mortality. Outcomes were selected based on feedback from patients with COPD and other stakeholders through a survey (see online supplementary appendix 3). The results of the survey appear in online supplementary appendix 4 ; these have been disseminated online. ${ }^{12}$

RCTs were included regardless of duration of follow-up, date of dissemination or publication status. Owing to feasibility constraints, we limited inclusion to English language articles; this has not been shown to bias meta-analysis estimates in the past. ${ }^{13}$ Owing to the large number of unpublished citations conference abstracts identified, we limited inclusion of unpublished data to abstracts from 2004 onwards because this is more likely to capture unreported data given the average time it takes for a RCT to be published. ${ }^{14}$ Authors were contacted for unpublished data obtained through study protocols and conference abstracts, as well as English translations of non-English articles.

\section{Information sources and literature search}

An experienced librarian conducted comprehensive literature searches in MEDLINE, EMBASE, and the Cochrane Central Register of Controlled Trials from inception until December 2013. The MEDLINE search was peer reviewed by another experienced librarian using the Peer Review of Electronic Search Strategies (PRESS) checklist, ${ }^{15}$ and revised as necessary (see online supplementary appendix 5). This was supplemented by manual searching of the reference lists of included studies and relevant reviews. ${ }^{1-9} 16$

\section{Study selection process}

Only when $>90 \%$ agreement was achieved through a training exercise, pairs of reviewers screened citations for inclusion, independently. The same process was followed for screening potentially relevant full-text articles. Conflicts were resolved by discussion or the involvement of a third reviewer (ACT or SES).

\section{Data items and data abstraction process}

After a calibration exercise, study characteristics (eg, year of conduct, sample size, setting), patient characteristics (eg, number of patients, age, mean age and SD) and the definitions of outcome definitions (eg, exacerbations (ie, number of patients with at least 1 exacerbation in the past year)) were abstracted independently by pairs of reviewers, independently. To ensure data integrity for the abstracted data, all data were verified by a third reviewer (LS, FY or AS). Since the Global Initiative for Chronic Obstructive Lung Disease (GOLD) criteria have changed over time, a clinician (SES) reviewed all of the included studies to ascertain the average COPD severity of the patients included in each RCT.

\section{Risk of bias and methodological quality appraisal process}

After a calibration exercise, pairs of reviewers independently assessed each of the included RCTs using the seven-item Cochrane Risk-of-Bias tool. ${ }^{17}$

\section{Synthesis}

A restricted maximum likelihood method ${ }^{18}$ and the $\mathrm{I}^{2}$ statistic $^{19}$ were used to estimate the magnitude and measure the between-study heterogeneity variance in meta-analysis, respectively. A random-effects network meta-analysis was conducted because we anticipated that the treatment effects were heterogeneous across the included RCTs. We assumed common between-study 
variance across treatment comparisons. As the included treatments are of the same nature, it is clinically reasonable to share a common heterogeneity parameter.

The treatment nodes of the network were selected based on input from clinicians, methodologists and statisticians on the team. Owing to the complexity of the analysis, we did not account for differences in doses and durations assuming that all impact the treatment effect equally. Specifically, when a study compared different doses of an intervention against another intervention, we included only the recommended dose in the analysis. As well, we conducted a specific drug analysis versus a drug class analysis, as this was what the policymakers associated with the ODPRN requested.

Before conducting the analyses, we assessed the transitivity assumption by exploring whether any systematic differences were prevalent in the distribution of potential treatment effect modifiers across treatment comparisons in the network. For each outcome, we examined the percentage of female participants (gender) in the RCTs and the risk-of-bias results. For the moderate-to-severe exacerbations outcome, we also examined RCTs with eligibility criteria focusing on patients who experienced an exacerbation in the past year and severity of COPD.

To evaluate the consistency assumption, we evaluated the network as a whole using the design-by-treatment interaction model. ${ }^{20}$ If inconsistency was prevalent, the loop-specific method was used to identify local inconsistency in parts of the network. ${ }^{21-23}$ When important inconsistency and/or heterogeneity were observed, we assessed for potential data abstraction errors, and if none were identified, we conducted a subgroup network meta-analysis on the potential treatment effect modifiers. We explored the effect of study duration in a random-effects meta-regression analysis for mortality and exacerbation outcomes, assuming a common fixed coefficient across treatment comparisons. To assess the robustness of our results, we conducted a sensitivity analysis limiting all of the analyses to studies with a low risk of bias according to the randomisation and allocation concealment components.

Summary treatment effects (ORs) from the network meta-analysis are presented, along with their respective 95\% CIs and 95\% predictive intervals (PrI). The PrI is more conservative than the CI and indicates the possible treatment effects, should an additional study become available. ${ }^{24}{ }^{25}$ It should be noted that a PrI is available only when the difference between the number of studies in the network and the number of available direct comparisons is greater than 2. A comparison-adjusted funnel plot was used to investigate the association between effect size and its SE (the latter is closely related to study size). If a relationship exists, this can be due to publication or related biases or due to systematic differences between small and large studies. ${ }^{26} \mathrm{~A}$ treatment hierarchy was also obtained using the SUrface under the Cumulative RAnking (SUCRA) curve analysis which allows the ranking of interventions according to the probability of being the most effective for each outcome (eg, most effective at reducing the risk of exacerbations, most harmful at increasing the risk of cardiovascularrelated mortality). ${ }^{27}$

\section{Model fit}

Random-effects meta-analyses were undertaken in $\mathrm{R}$ V.3.1.2 using the meta package, ${ }^{28} 29$ while random-effects network meta-analyses were conducted in STATA V.13.1 using the mumeta command. ${ }^{30} 31$ We implemented network meta-regression analyses on the study duration variable in OpenBUGS V.3.2.3, ${ }^{32}$ using 100000 simulations with a thinning rate of 10 after discarding the first 30000 iterations. Convergence was assessed by visual inspection of the mixing of two chains with different initial values. We assumed a vague prior for the coefficient parameter $\left(\mathrm{N}\left(0,10^{4}\right)\right)$ and an informative prior for the between-study variance, as suggested by Turner et $a l^{33}\left(\tau^{2} \sim \log \mathrm{N}\left(-2.13,1.58^{2}\right)\right)$.

\section{RESULTS}

\section{Literature search}

The literature search yielded a total of 2447 titles and abstracts (figure 1). Of these, 980 articles were potentially relevant and their full text was reviewed. Subsequently, 203 RCTs providing data on 208 RCTs (some trials reported the results from more than one study) plus 58 companion reports fulfilled our eligibility criteria and were included. The list of the included studies and their references can be found in online supplementary appendix 6 . Twenty of the included studies were unpublished.

\section{Study and patient characteristics}

The year of publication ranged from 1989 to 2014 (table 1, online supplementary appendix 7). Most RCTs were multicentre trials conducted across numerous countries. The duration of treatment with long-acting inhaled agents ranged from $9 \mathrm{~h}$ to almost 4 years. Most of the RCTs reported moderate-to-severe COPD exacerbations (54\%) and mortality (46\%). The presence of serious arrhythmia was the least frequently reported outcome ( $15 \%$ of studies).

The total number of patients across the RCTs was 134692 , with an average of 648 patients per trial (table 2, see online supplementary appendix 8 ). The severity of COPD was most commonly moderate-tosevere or moderate-to-very severe $(61 \%)$ in nature. The percentage of females in the included studies ranged from $0 \%$ to $58 \%$.

\section{Risk of bias}

Across the included RCTs, the majority had an unclear random sequence generation $(63 \%)$ and unclear allocation concealment (84\%) risk of bias (figure 2, see online supplementary appendix 9). In addition, the majority had an unclear risk of bias (55\%) related to 
Figure 1 Study flow diagram details the flow of information through the different phases of the review; maps out the number of records identified, included and excluded, and the reasons for their exclusion (COPD, chronic obstructive pulmonary disease).

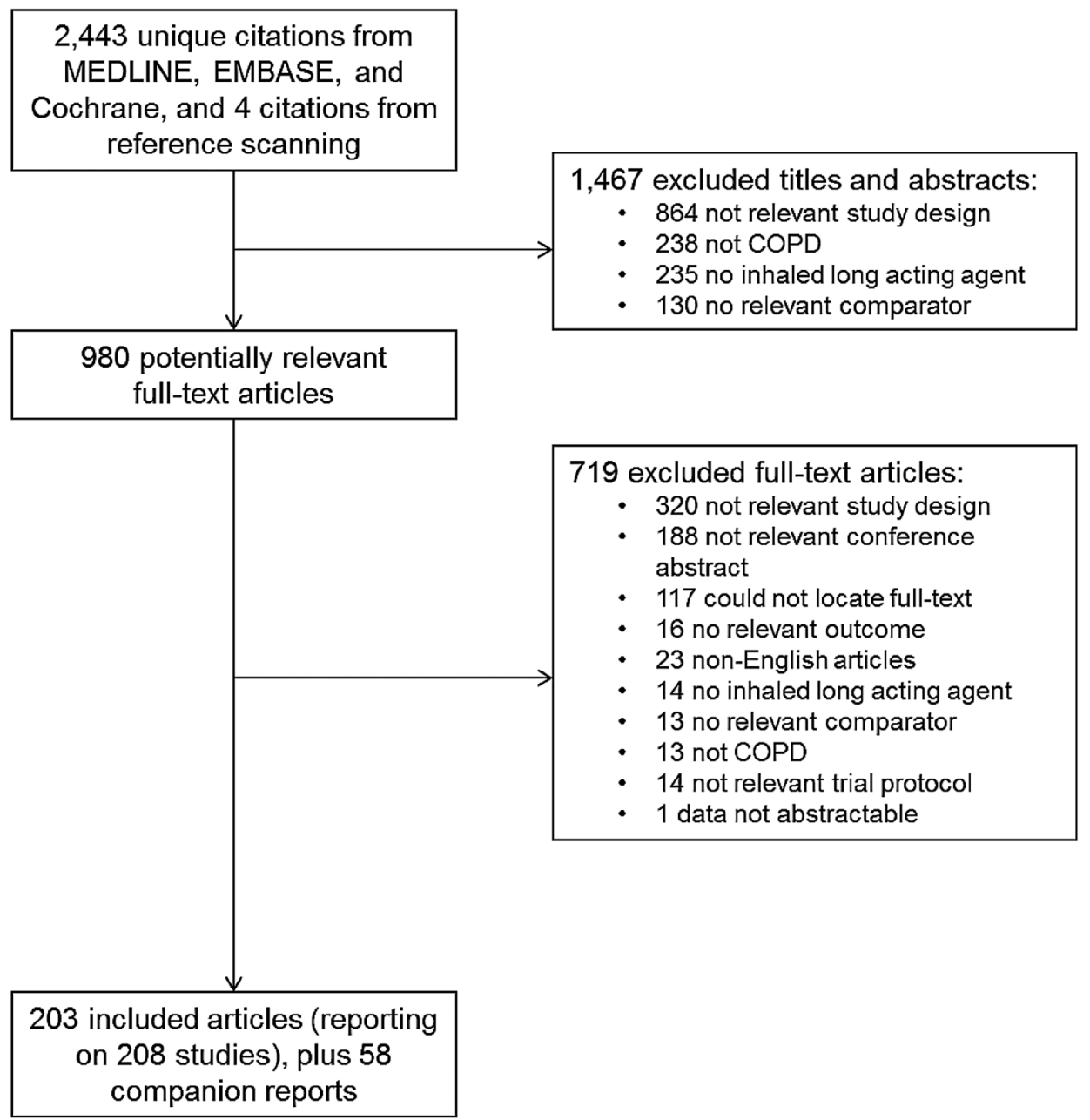

selective outcome reporting, as the outcomes reported in the trial protocols differed from those reported in the final publication. Finally, many of the RCTs had a high $(52 \%)$ or unclear $(39 \%)$ risk of bias due to the 'other bias' item, mainly owing to the potential for funding bias as many studies were funded by a pharmaceutical company and included study authors who were employed by the drug manufacturer. Finally, visual inspection of the comparison-adjusted funnel plots showed that there was no evidence for small-study effects and publication bias across all analyses.

\section{Moderate-to-severe exacerbations}

A network meta-analysis was attempted with 112 RCTs including 77749 patients and 26 inhaled treatments for patients presenting with moderate-to-severe exacerbations (ie, worsening of COPD symptoms that may require hospitalisation, emergency department visits, treatment with oral steroids and/or antibiotics, use of rescue medication, unscheduled walk-in clinic/healthcare provider visits). However, significant inconsistency was observed between direct and indirect evidence $\left(\chi^{2}=80.74\right.$, degrees of freedom $=51, p=0.005$, heterogeneity variance $=0.01$ ). As such, a subgroup network meta-analysis was conducted including only those trials with patients who had experienced an exacerbation in the past year (figure 3A). For this analysis, 20 RCTs were included with 26141 patients and 17 treatments; there was no evidence of statistical inconsistency $\left(\chi^{2}=3.37\right.$, degrees of freedom $=4, p=0.50$, heterogeneity variance $=0.00)$. Of the 136 treatment comparisons in the network meta-analysis model, 32 were statistically significant (table 3, see online supplementary appendices 10 and 11) and 8 of these were more effective than placebo in reducing the risk of moderate-to-severe exacerbations: tiotropium, salmeterol, indacaterol, budesonide/formoterol, fluticasone/salmeterol, indacaterol/glycopyrronium, tiotropium/fluticasone/salmeterol and tiotropium/ budesonide/formoterol. The most effective were tiotropium/budesonide/formoterol (99\% probability of being the most effective in reducing exacerbations) and indacaterol/glycopyrronium $(86 \%)$ according to the SUCRA curves (see online supplementary appendix 12).

A sensitivity analysis was conducted with studies at a low risk of randomisation and allocation concealment biases. Based on 25 RCTs, 20 treatments and 33211 patients meeting these criteria, 190 treatment comparisons were made in the network meta-analysis model. Twenty-four of these were statistically significant, including three that reduced the risk of moderate-to-severe 
Table 1 Study characteristics

\begin{tabular}{|c|c|c|}
\hline Characteristic & $\begin{array}{l}\text { Number of } \\
\text { studies* }^{*} \\
(n=208)\end{array}$ & $\begin{array}{l}\text { Percentage } \\
\text { of studies }\end{array}$ \\
\hline \multicolumn{3}{|l|}{ Year of publication } \\
\hline 1989-1994 & 3 & 1.4 \\
\hline 1995-1999 & 15 & 7.2 \\
\hline $2000-2004$ & 35 & 16.8 \\
\hline 2005-2009 & 68 & 32.7 \\
\hline 2010-2014 & 87 & 41.8 \\
\hline \multicolumn{3}{|l|}{ Geographic region } \\
\hline Europe & 72 & 34.6 \\
\hline Multicontinent & 44 & 21.2 \\
\hline North America & 36 & 17.3 \\
\hline Multicountry (not specified) & 24 & 11.5 \\
\hline Asia & 20 & 9.6 \\
\hline Not reported & 9 & 4.3 \\
\hline Africa & 1 & 0.5 \\
\hline Australia & 1 & 0.5 \\
\hline South America & 1 & 0.5 \\
\hline \multicolumn{3}{|l|}{ Setting } \\
\hline Single centre & 32 & 15.4 \\
\hline Multicentre & 152 & 73.1 \\
\hline Not reported & 24 & 11.5 \\
\hline \multicolumn{3}{|l|}{ Duration of follow-upt } \\
\hline 0 to $\leq 6$ & 45 & 21.6 \\
\hline$>6$ to $\leq 12$ & 52 & 25 \\
\hline$>12$ to $\leq 24$ & 35 & 16.8 \\
\hline$>24$ to $\leq 48$ & 19 & 9.1 \\
\hline$>48$ to $\leq 72$ & 39 & 18.8 \\
\hline$>72$ to $\leq 96$ & 2 & 1 \\
\hline$>96$ to $\leq 120$ & 6 & 2.9 \\
\hline$>120$ weeks & 9 & 4.3 \\
\hline Not reported & 1 & 0.5 \\
\hline \multicolumn{3}{|l|}{ Outcomes examined: frequency $\ddagger$} \\
\hline Efficacy-exacerbations & 112 & 53.8 \\
\hline Efficacy-mortality & 95 & 45.7 \\
\hline $\begin{array}{l}\text { Harm-cardiovascular- } \\
\text { related mortality }\end{array}$ & 46 & 22.1 \\
\hline Harm-pneumonia & 54 & 26.0 \\
\hline Harm-arrhythmia & 32 & 15.4 \\
\hline
\end{tabular}

exacerbations compared with placebo; fluticasone, indacaterol/tiotropium and indacaterol/glycopyrronium (figure 4). The most effective agent was fluticasone according to the SUCRA curves $(96 \%)$, which was followed by indacaterol/glycopyrronium (80\%), and mometasone/formoterol (80\%). A statistically significant association was not observed in our metaregression analysis conducted using the study duration as a covariate (estimated coefficient: 1.01 (95\% credible interval (CrI) $0.41,2.41)$, heterogeneity variance $=0.02$ ) .

\section{Mortality}

Six studies were excluded from the analysis because they reported 0 events in all relevant treatment arms. ${ }^{34-39}$
Table 2 Patient characteristics

Total number of patients 134692

Mean sample size 648

Mean \%, female $27.7 \%$

\begin{tabular}{lrc}
\hline Characteristic & $\begin{array}{l}\text { Number of } \\
\text { studies* } \\
(\mathbf{n}=\mathbf{2 0 8})\end{array}$ & $\begin{array}{c}\text { Percentage } \\
\text { of studies }\end{array}$ \\
\hline Age category & & \\
$\quad$ Adult and elderly $(\geq 18)$ & 195 & 93.8 \\
Adult (18-64) & 4 & 1.9 \\
Elderly ( $\geq 65)$ & 0 & 0.0 \\
NR & 9 & 4.3 \\
Severity of COPD & & \\
$\quad$ Mild to moderate & 10 & 4.8 \\
Mild to severe & 9 & 4.3 \\
Mild to very severe & 8 & 3.8 \\
Moderate & 7 & 3.4 \\
Moderate to severe & 60 & 28.9 \\
Moderate to very severe & 67 & 32.2 \\
Severe & 5 & 2.4 \\
Severe to very severe & 6 & 2.9 \\
Stable (severity NR) & 5 & 2.4 \\
NR & 31 & 14.9 \\
Female, \% & & \\
0-25 & 90 & 43.3 \\
26-50 & 104 & 50 \\
51-100 & 4 & 1.9 \\
Not reported & 10 & 4.8 \\
\hline *Includes unpublished studies. & \\
COPD, chronic obstructive pulmonary disease; NR, not reported. &
\end{tabular}

As such, a network meta-analysis was conducted with 88 RCTs, 28 treatments and 97526 patients (figure 3B). There was no evidence of statistical inconsistency $\left(\chi^{2}=31.44\right.$, degrees of freedom $=50, p$ value $=0.98$, heterogeneity variance $=0.00$ ). Of the 378 treatment comparisons in the network meta-analysis model, only three were statistically significant. Fluticasone/salmeterol combination therapy resulted in a reduced risk of mortality compared with placebo, formoterol and fluticasone alone (table 3 , see online supplementary appendices 10 and 11). The most effective agent in having a reduced risk of mortality was fluticasone/salmeterol as determined by the SUCRA curves $(71 \%$; see online supplementary appendix 12).

A sensitivity analysis was conducted with studies at a low risk of randomisation generation and allocation concealment biases. Based on 23 RCTs, 21 treatments and 33624 patients, 210 treatment comparisons were made in the network meta-analysis model. Four of these were statistically significant, as follows: fluticasone/salmeterol was superior to placebo, salmeterol alone, tiotropium and vilanterol (figure 5). The most effective agent was the inhaled combination of fluticasone/salmeterol (90\%) according to the SUCRA curves. A statistically significant association was not observed in our metaregression analysis conducted using the study duration as a covariate (estimated coefficient 1.00 (95\% CrI 0.88, $1.14)$, heterogeneity variance $=0.03$ ) . 


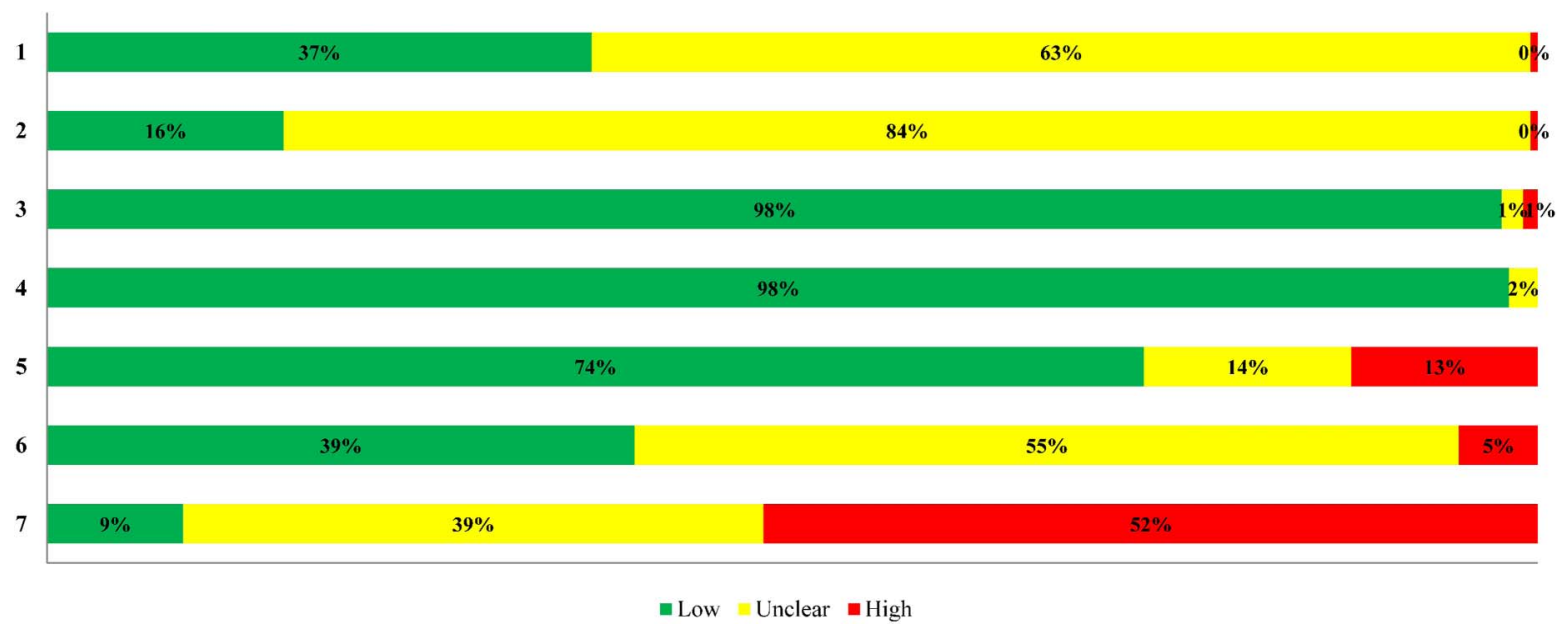

Figure 2 Risk of bias appraisal results. High, high risk of bias; low, low risk of bias; unclear, unclear risk of bias. Items: 1. Random sequence generation 2. Allocation concealment 3. Blinding of participants and personnel 4. Blinding of outcome assessment 5 . Incomplete outcome data 6 . Selective reporting 7. Other bias.

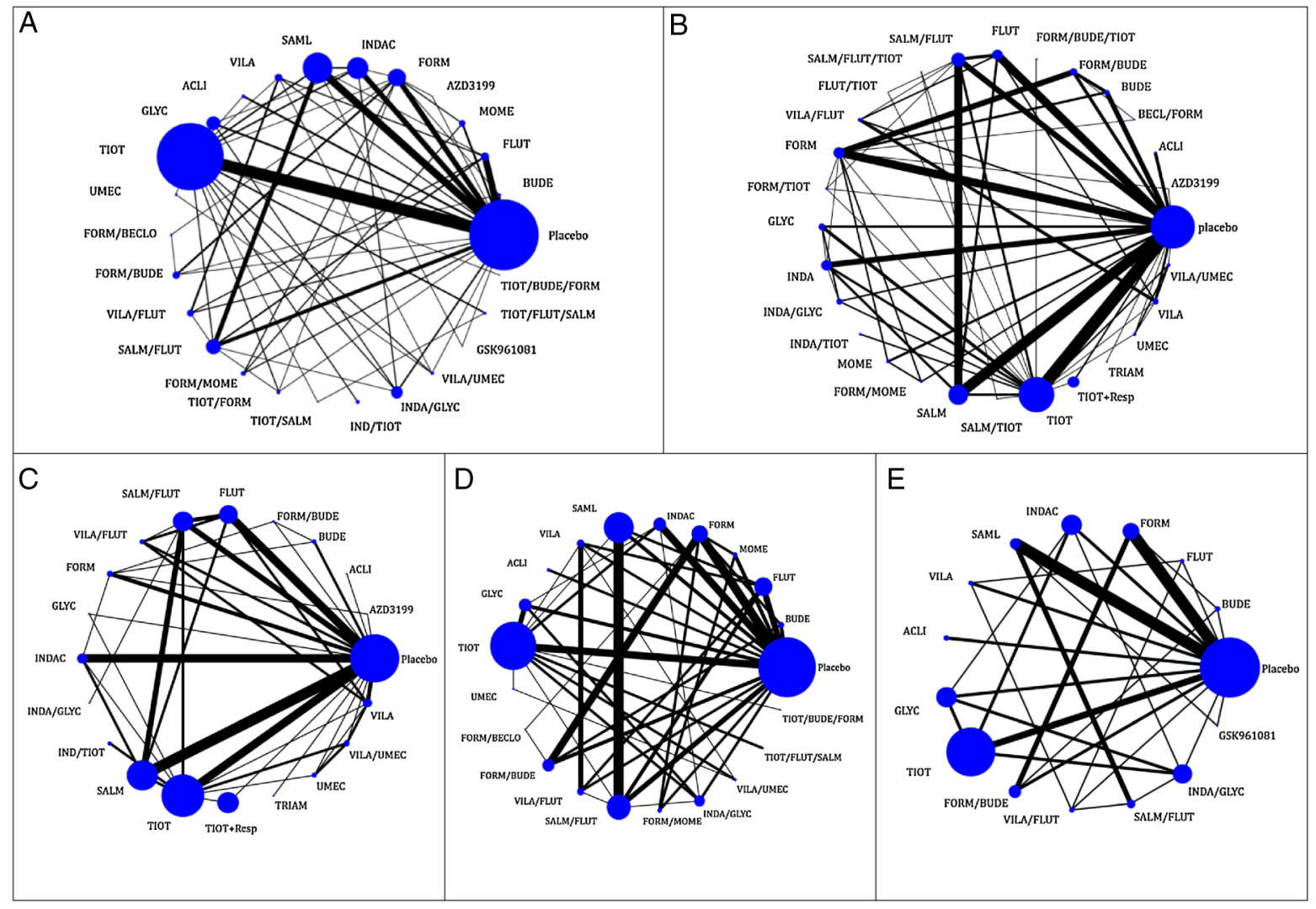

Figure 3 Network meta-analysis plots. (A) Exacerbation, (B) mortality, (C) cardiovascular-related mortality, (D) pneumonia and (E) serious arrhythmia. Nodes are proportional to the number of patients included in the corresponding treatments, and edges are weighted according to the number of studies included in the respective comparisons. BECL, beclomethasone; BUDE, budesonide; FLUT, fluticasone; MOME, mometasone; TRIAM, triamcinolone acetonide; AZD3199, AZD3199 (ultra LABA); FORM, formoterol; INDAC, indacaterol ; SALM, SAML, salmeterol; VILA, vilanterol; ACLI, aclidinium bromide; GLYC, glycopyrronium bromide; DAROT, darotropium bromide; TIOT, tiotropium; UMEC, umeclidinium; FORM/BECLO, BECL/FORM, formoterol/beclomethasone; FORM/BUDE, formoterol/budesonide; VILA/FLUT, vilanterol/fluticasone; SALM/FLUT, salmeterol/ fluticasone/; FORM/MOME, formoterol/mometasone; TIOT/BUDE, tiotropium/budesonide; TIOT/FLUT, FLUT/TIOT, tiotropium/ fluticasone; TIOT/FORM, FORM/TIOT, tiotropium/formoterol; TIOT/SALM, tiotropium/salmeterol; IND/TIOT, indacaterol/tiotropium; INDA/GLYC, indacaterol/glycopyrronium; VILA/UMEC, vilanterol/umeclidinium; GSK961081, GSK961081; FORM/IPRATR, formoterol+ipratropium bromide; TIOT/FLUT/SALM, tiotropium/fluticasone/salmeterol; TIOT/BUDE/FORM, FORM/BUDE/TIOT, tiotropium/budesonide/formoterol; BUDE/FORM/IPRATR, budesonide/formoterol/ipratropium bromide; TIOT+Resp, Tiotropium Respimat (Soft Mist Inhaler). 
Table 3 Statistically significant network meta-analysis results

\begin{tabular}{|c|c|c|c|c|c|c|}
\hline Treatment comparison & $\begin{array}{l}\text { NMA } \\
\text { estimate } \\
\text { OR } \\
(95 \% \mathrm{Cl})\end{array}$ & $\mathbf{C l}$ & $\begin{array}{l}\text { MA } \\
\text { estimate } \\
\text { OR } \\
(95 \% \mathrm{CI})\end{array}$ & $\mathbf{C l}$ & $\begin{array}{l}\text { Number of } \\
\text { studies } \\
\text { (Number of } \\
\text { patients) }\end{array}$ & $\begin{array}{l}\text { MA } \\
\text { Heterogeneity } \\
\text { variance }\end{array}$ \\
\hline \multicolumn{7}{|c|}{ Exacerbation past year-20 studies, 17 treatments, 26141 patients } \\
\hline FLUT/SALM vs SALM & 0.85 & $0.75-0.97$ & 0.82 & $0.70-0.95$ & $4(2784)$ & 0.00 \\
\hline TIOT vs INDAC & 0.83 & 0.72 to 0.96 & 0.83 & 0.72 to 0.96 & 1 (3439) & - \\
\hline TIOT vs SALM & 0.82 & 0.73 to 0.93 & 0.84 & 0.76 to 0.92 & $1(7376)$ & - \\
\hline SALM vs placebo & 0.79 & 0.64 to 0.97 & 0.80 & 0.58 to 1.09 & $1(634)$ & - \\
\hline INDAC vs placebo & 0.78 & 0.61 to 1.00 & & & & \\
\hline BUDE/FORM vs FORM & 0.76 & 0.64 to 0.91 & 0.76 & 0.62 to 0.93 & $4(3080)$ & 0.01 \\
\hline FLUT/F vs VILA & 0.75 & 0.62 to 0.92 & 0.75 & 0.61 to 0.94 & $2(1624)$ & 0.00 \\
\hline INDAC/GLYC vs TIOT & 0.74 & 0.60 to 0.91 & 0.74 & 0.60 to 0.91 & $1(1466)$ & - \\
\hline INDAC/GLYC vs FLUT/SALM & 0.71 & 0.55 to 0.92 & & & & \\
\hline FLUT/SALM vs Placebo & 0.67 & 0.53 to 0.85 & & & & \\
\hline TIOT vs Placebo & 0.65 & 0.53 to 0.79 & 0.64 & 0.50 to 0.83 & $1(1003)$ & - \\
\hline BUDE/FORM vs placebo & 0.64 & 0.45 to 0.91 & 0.55 & 0.36 to 0.83 & $1(519)$ & - \\
\hline INDAC/GLYC vs GLYC & 0.63 & 0.51 to 0.78 & 0.63 & 0.51 to 0.77 & $1(1469)$ & - \\
\hline INDAC/GLYC vs INDAC & 0.62 & 0.48 to 0.79 & & & & \\
\hline INDAC/GLYC vs SALM & 0.61 & 0.48 to 0.78 & & & & \\
\hline TIOT/FLUT/SALM vs placebo & 0.58 & 0.35 to 0.96 & & & & \\
\hline INDAC/GLYC vs FORM & 0.57 & 0.36 to 0.90 & & & & \\
\hline TIOT/BUDE/FORM vs INDAC/GLYC & 0.48 & 0.28 to 0.83 & & & & \\
\hline INDAC/GLYC vs placebo & 0.48 & 0.36 to 0.64 & & & & \\
\hline TIOT/BUDE/FORM vs TIOT/FLUT/ & 0.40 & 0.21 to 0.80 & & & & \\
\hline SALM & & & & & & \\
\hline TIOT/BUDE/FORM vs BUDE/FORM & 0.36 & 0.19 to 0.69 & & & & \\
\hline TIOT/BUDE/FORM vs TIOT & 0.36 & 0.22 to 0.59 & 0.36 & 0.22 to 0.59 & $1(660)$ & - \\
\hline TIOT/BUDE/FORM vs FLUT/SALM & 0.35 & 0.21 to 0.58 & & & & \\
\hline TIOT/BUDE/FORM vs TIOT/SALM & 0.33 & 0.17 to 0.65 & & & & \\
\hline TIOT/BUDE/FORM vs BECL/FORM & 0.32 & 0.15 to 0.65 & & & & \\
\hline TIOT/BUDE/FORM vs BUDE & 0.31 & 0.16 to 0.60 & & & & \\
\hline TIOT/BUDE/FORM vs GLYC & 0.30 & 0.18 to 0.52 & & & & \\
\hline TIOT/BUDE/FORM vs INDAC & 0.30 & 0.18 to 0.50 & & & & \\
\hline TIOT/BUDE/FORM vs SALM & 0.30 & 0.18 to 0.49 & & & & \\
\hline TIOT/BUDE/FORM vs FLUT & 0.29 & 0.14 to 0.60 & & & & \\
\hline TIOT/BUDE/FORM vs FORM & 0.28 & 0.15 to 0.52 & & & & \\
\hline TIOT/BUDE/FORM vs placebo & 0.23 & 0.14 to 0.40 & & & & \\
\hline \multirow{2}{*}{\multicolumn{3}{|c|}{$\begin{array}{l}\text { Between-study heterogeneity variance for NMA } \\
\text { Design-by-treatment interaction model for inconsistency } \chi^{2} \\
\text { (df, } p \text { value, heterogeneity) }\end{array}$}} & \multicolumn{4}{|l|}{0.00} \\
\hline & & & 3.37 & $498,0.00)$ & & \\
\hline \multicolumn{7}{|c|}{ Mortality overall-88 studies, 28 treatments, 97526 patients } \\
\hline FORM vs FLUT/SALM & 1.64 & 1.01 to 2.67 & & & & 0.00 \\
\hline FLUT/SALM vs Placebo & 0.78 & 0.63 to 0.96 & 0.81 & 0.66 to 1.00 & $6(4852)$ & 0.00 \\
\hline FLUT/SALM vs FLUT & 0.75 & 0.60 to 0.94 & 0.76 & 0.62 to 0.93 & $3(3752)$ & 0.00 \\
\hline \multirow{2}{*}{\multicolumn{3}{|c|}{$\begin{array}{l}\text { Between-study heterogeneity variance for NMA } \\
\text { Design-by-treatment interaction model for inconsistency } \chi^{2} \\
\text { (df, p value, heterogeneity) }\end{array}$}} & \multicolumn{4}{|l|}{0.00} \\
\hline & & & $31.44(50$ & $0.982,0.00)$ & & \\
\hline \multicolumn{7}{|c|}{ Cardiovascular-related mortality-37 studies, 20 treatments, 55156 patients } \\
\hline TIOT+Resp vs SALM & 2.32 & 1.38 to 3.88 & & \multirow{3}{*}{0.46 to 3.81} & & \\
\hline TIOT vs SALM & 2.00 & 1.23 to 3.26 & \multirow[t]{3}{*}{1.32} & & \multirow[t]{2}{*}{$1(7798)$} & \multirow[t]{2}{*}{-} \\
\hline TIOT+Resp vs FLUT/SALM & 1.87 & 1.14 to 3.06 & & & & \\
\hline TIOT+Resp vs FLUT & 1.75 & 1.04 to 2.94 & & & & \\
\hline TIOT vs FLUT/SALM & 1.61 & 1.02 to 2.56 & 2.12 & 0.95 to 4.72 & $1(1448)$ & - \\
\hline SALM vs placebo & 0.63 & 0.45 to 0.88 & 0.60 & 0.42 to 0.87 & $4(5171)$ & 0.00 \\
\hline \multirow{2}{*}{\multicolumn{3}{|c|}{$\begin{array}{l}\text { Between-study heterogeneity variance for NMA } \\
\text { Design-by-treatment interaction model for inconsistency } \chi^{2} \\
\text { (df, p value, heterogeneity) }\end{array}$}} & \multirow{2}{*}{\multicolumn{2}{|c|}{$\begin{array}{l}0.00 \\
11.79(27,0.995,0.00)\end{array}$}} & & \\
\hline & & & & & & \\
\hline
\end{tabular}


Table 3 Continued

\begin{tabular}{|c|c|c|c|c|c|c|}
\hline Treatment comparison & $\begin{array}{l}\text { NMA } \\
\text { estimate } \\
\text { OR } \\
(95 \% \mathrm{Cl})\end{array}$ & $\mathrm{Cl}$ & $\begin{array}{l}\text { MA } \\
\text { estimate } \\
\text { OR } \\
(95 \% \mathrm{Cl})\end{array}$ & $\mathbf{C l}$ & $\begin{array}{l}\text { Number of } \\
\text { studies } \\
\text { (Number of } \\
\text { patients) }\end{array}$ & $\begin{array}{l}\text { MA } \\
\text { Heterogeneity } \\
\text { variance }\end{array}$ \\
\hline \multicolumn{7}{|c|}{ Pneumonia-54 studies, 21 treatments, 61551 patients } \\
\hline FLUT/VILA vs ACLI & 3.15 & 1.07 to 9.24 & & & & \\
\hline FLUT/VILA vs BUDE & 2.83 & 1.10 to 7.25 & & & & \\
\hline FLUT/SALM vs ACLI & 2.81 & 1.30 to 6.07 & & & & \\
\hline FLUT/VILA vs GLYC & 2.59 & 1.09 to 6.18 & & & & \\
\hline FLUT/SALM vs BUDE & 2.52 & 1.44 to 4.43 & & & & \\
\hline FLUT/SALM vs GLYC & 2.31 & 1.47 to 3.64 & & & & \\
\hline FLUT/VILA vs TIOT & 2.25 & 1.02 to 4.96 & & & & \\
\hline FLUT vs BUDE & 2.21 & 1.25 to 3.92 & & & & \\
\hline FLUT/SALM vs FORM & 2.09 & 1.29 to 3.37 & & & & \\
\hline FLUT/SALM vs TIOT & 2.00 & 1.52 to 2.64 & 2.20 & 1.33 to 3.62 & $1(1323)$ & - \\
\hline FLUT/SALM vs INDAC & 1.95 & 1.20 to 3.17 & & & & \\
\hline FLUT/SALM vs placebo & 1.90 & 1.53 to 2.34 & 1.75 & 1.44 to 2.13 & $4(3872)$ & $<0.0001$ \\
\hline FLUT/VILA vs VILA & 1.87 & 1.18 to 2.96 & 1.90 & 1.20 to 3.01 & $4(2442)$ & 0.00 \\
\hline FLUT/SALM vs SALM & 1.70 & 1.38 to 2.09 & 1.69 & 1.40 to 2.04 & $8(7613)$ & 0.00 \\
\hline FLUT vs placebo & 1.66 & 1.32 to 2.08 & 1.60 & 1.32 to 1.95 & $5(4258)$ & 0.00 \\
\hline SALM vs FLUT & 0.67 & 0.54 to 0.84 & 0.68 & 0.56 to 0.83 & $2(3174)$ & 0.00 \\
\hline INDAC vs FLUT & 0.58 & 0.36 to 0.95 & & & & \\
\hline TIOT vs FLUT & 0.57 & 0.43 to 0.75 & & & & \\
\hline FORM vs FLUT & 0.55 & 0.33 to 0.90 & & & & \\
\hline INDAC/GLYC vs FLUT & 0.51 & 0.31 to 0.85 & & & & \\
\hline GLYC vs FLUT & 0.49 & 0.31 to 0.78 & & & & \\
\hline INDAC/GLYC vs FLUT/SALM & 0.45 & 0.27 to 0.75 & 0.11 & 0.01 to 2.09 & $1(522)$ & - \\
\hline ACLI vs FLUT & 0.41 & 0.19 to 0.88 & & & & \\
\hline INDAC/GLYC vs FLUT/VILA & 0.40 & 0.16 to 0.98 & & & & \\
\hline \multirow{2}{*}{\multicolumn{3}{|c|}{$\begin{array}{l}\text { Between-study heterogeneity variance for NMA } \\
\text { Design-by-treatment interaction model for inconsistency } \chi^{2} \\
\text { (d.f., p value, heterogeneity) }\end{array}$}} & \multicolumn{4}{|l|}{0.01} \\
\hline & & & \multicolumn{4}{|c|}{$34.33(31,0.311,0.00)$} \\
\hline
\end{tabular}

\section{Cardiovascular-related mortality}

Nine studies were excluded from the analysis of cardiovascular-related mortality (including cardiac arrest, aortic aneurysm and myocardial infarction) because they reported 0 events in all relevant treatment arms. ${ }^{34-42}$ As such, a network meta-analysis was conducted including 37 RCTs, 20 treatments and 55156 patients (figure 3C). There was no evidence of statistical inconsistency $\left(\chi^{2}=13.05\right.$, degrees of freedom $=24, p=0.97$, heterogeneity variance $=0.00$ ). A total of 190 treatment comparisons were made in the network meta-analysis model and the following six were statistically significant: salmeterol had a decreased risk of cardiovascular-related mortality versus placebo, tiotropium (Handihaler), and tiotropium (Soft Mist Inhaler). In addition, fluticasone was superior to tiotropium (Soft Mist Inhaler); and the salmeterol/fluticasone combination was superior to both tiotropium (Handihaler) and tiotropium (Soft Mist Inhaler; table 3, see online supplementary appendices 10 and 11). None of these treatment comparisons remained statistically significant according to the PrI, except for salmeterol versus tiotropium (Soft Mist
Inhaler). According to the SUCRA curves (see online supplementary appendix 12), the following were the most harmful: triamcinolone acetonide $(81 \%$ probability of being the most harmful because of a greater risk of cardiovascular-related mortality), formoterol/budesonide $(73 \%)$ and vilanterol/umeclidinium (73\%). However, these particular SUCRA results should be interpreted with caution, as some of these interventions were not statistically different from the other agents according to the effect sizes and $95 \%$ CIs.

A sensitivity analysis was conducted including only studies with a low risk of bias for cardiovascular-related mortality with 11 RCTs, 12 treatments 16443 patients, and 66 treatment comparisons; none of the results were statistically significant.

\section{Pneumonia}

One study was excluded from the analysis for reporting 0 events in all treatment arms. ${ }^{39}$ As such, 54 RCTs including 21 treatments and 61551 patients were included in a network meta-analysis for pneumonia (figure 3D). There was no evidence of statistical inconsistency $\left(\chi^{2}=34.33\right.$, 


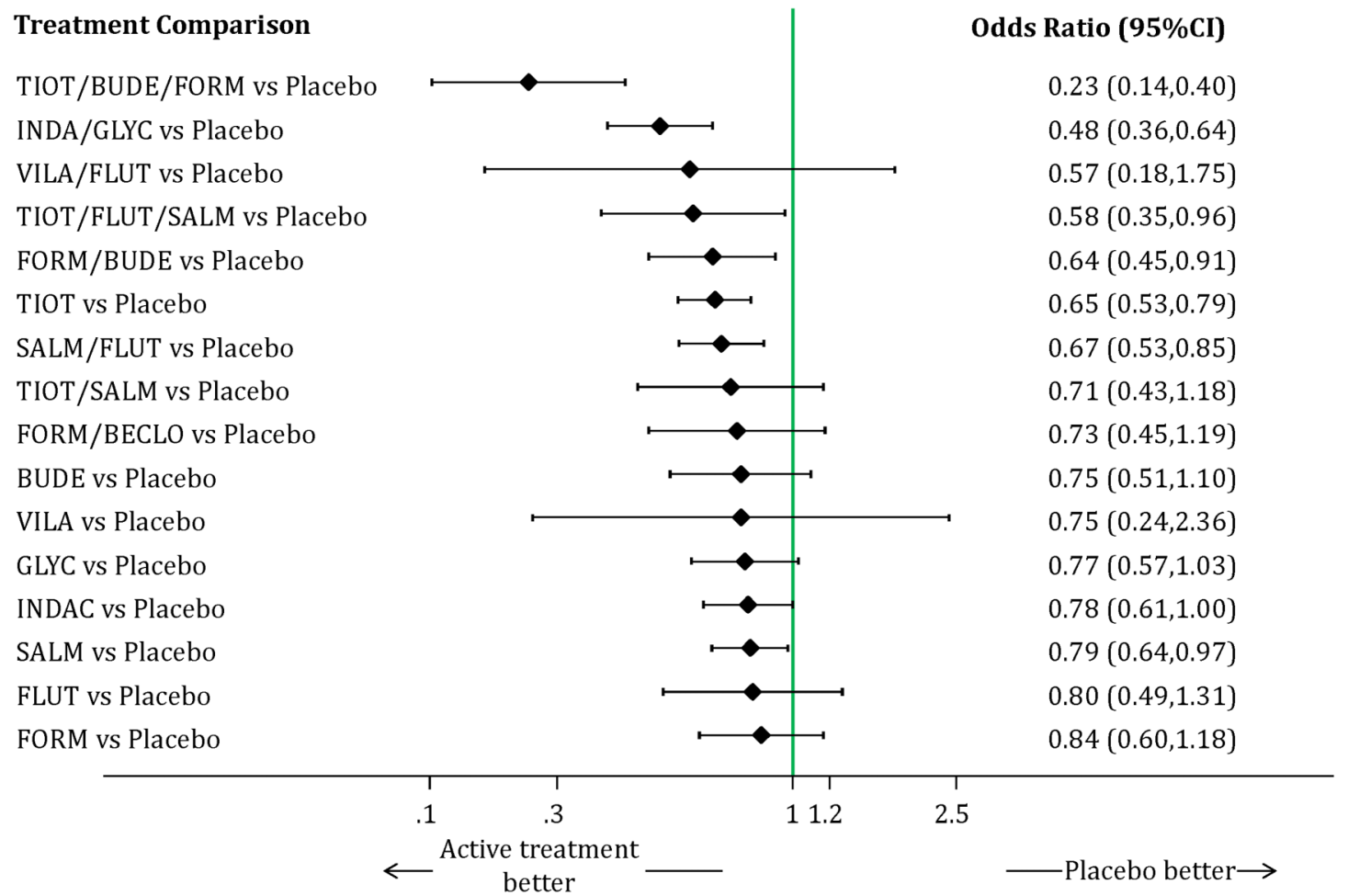

Figure 4 Moderate-to-severe exacerbations for patients who experienced an exacerbation in the past year Network metaanalysis results for treatments compared with placebo. The black horizontal lines represent the $95 \% \mathrm{Cls}$ of the summary treatment effects BUDE, budesonide; FLUT, fluticasone; FORM, formoterol; INDAC, indacaterol; SALM, salmeterol; VILA, vilanterol; GLYC, glycopyrronium bromide; TIOT, tiotropium; FORM/BECLO, formoterol/beclomethasone; FORM/BUDE, formoterol/budesonide; VILA/FLUT, vilanterol/fluticasone; SALM/FLUT, salmeterol/fluticasone; TIOT/SALM, tiotropium/salmeterol; INDA/GLYC, indacaterol/glycopyrronium; TIOT/FLUT/SALM, tiotropium/fluticasone/salmeterol; TIOT/BUDE/FORM, tiotropium/ budesonide/formoterol.

degrees of freedom $=31, \mathrm{p}=0.31$, heterogeneity variance $=0.00)$. A total of 210 treatment comparisons were made in the network meta-analysis model; 24 were statistically significant (table 3, see online supplementary appendices 11 and 12). Of these, two agents had a greater risk of pneumonia versus placebo; fluticasone and fluticasone/salmeterol. The following were the most harmful agents because they had a greater risk of pneumonia: fluticasone/salmeterol (SUCRA=89\%), fluticasone/vilanterol (SUCRA $=88 \%)$ and fluticasone (SUCRA=82\%; see online supplementary appendix 12).

A sensitivity analysis was conducted including only studies with a low risk of bias with 19 RCTs, 18 treatments and 28763 patients. There were 153 treatment comparisons in the network meta-analysis model and 17 were statistically significant (see online supplementary appendix 13) including two that were more harmful than placebo because they had a greater risk of pneumonia: budesonide/formoterol and fluticasone/salmeterol. The most harmful agents were budesonide/formoterol (SUCRA=94\%), beclomethasone/ formoterol $(\mathrm{SUCRA}=89 \%)$ and fluticasone $/$ salmeterol $(\mathrm{SUCRA}=78 \%)$.

\section{Serious arrhythmia}

Five studies were excluded from the analysis because they reported 0 events in all treatment arms. ${ }^{39} 43-46$ As such, a network meta-analysis was conducted including 26 RCTs, 12 treatments and 27407 patients (figure 3E) for serious arrhythmia (including atrial fibrillation and tachycardia). None of the 66 treatment comparisons were statistically significant (see online supplementary appendices 11 and 12) and no evidence of statistical inconsistency was observed $\left(\chi^{2}=3.06\right.$, degrees of freedom $=11, \mathrm{p}=0.99$, heterogeneity variance $=0.36$ ). The same results were observed in a sensitivity analysis involving six studies at low risk of bias with 7 treatments, 13060 patients, and 22 treatment comparisons.

\section{DISCUSSION}

For risk of a moderate-to-severe COPD exacerbation, we could not complete a network meta-analysis overall because the data were inconsistent. We were able to present results of our network meta-analysis for moderate-to-severe COPD exacerbation among patients who had experienced an exacerbation in the past year. We found that tiotropium/budesonide/formoterol and indacaterol/glycopyrronium combinations were the most effective inhaled agents at minimising the risk of a moderate-to-severe COPD exacerbation. Furthermore, we performed sensitivity analysis for moderate-to-severe exacerbations which included all studies rated as scoring 


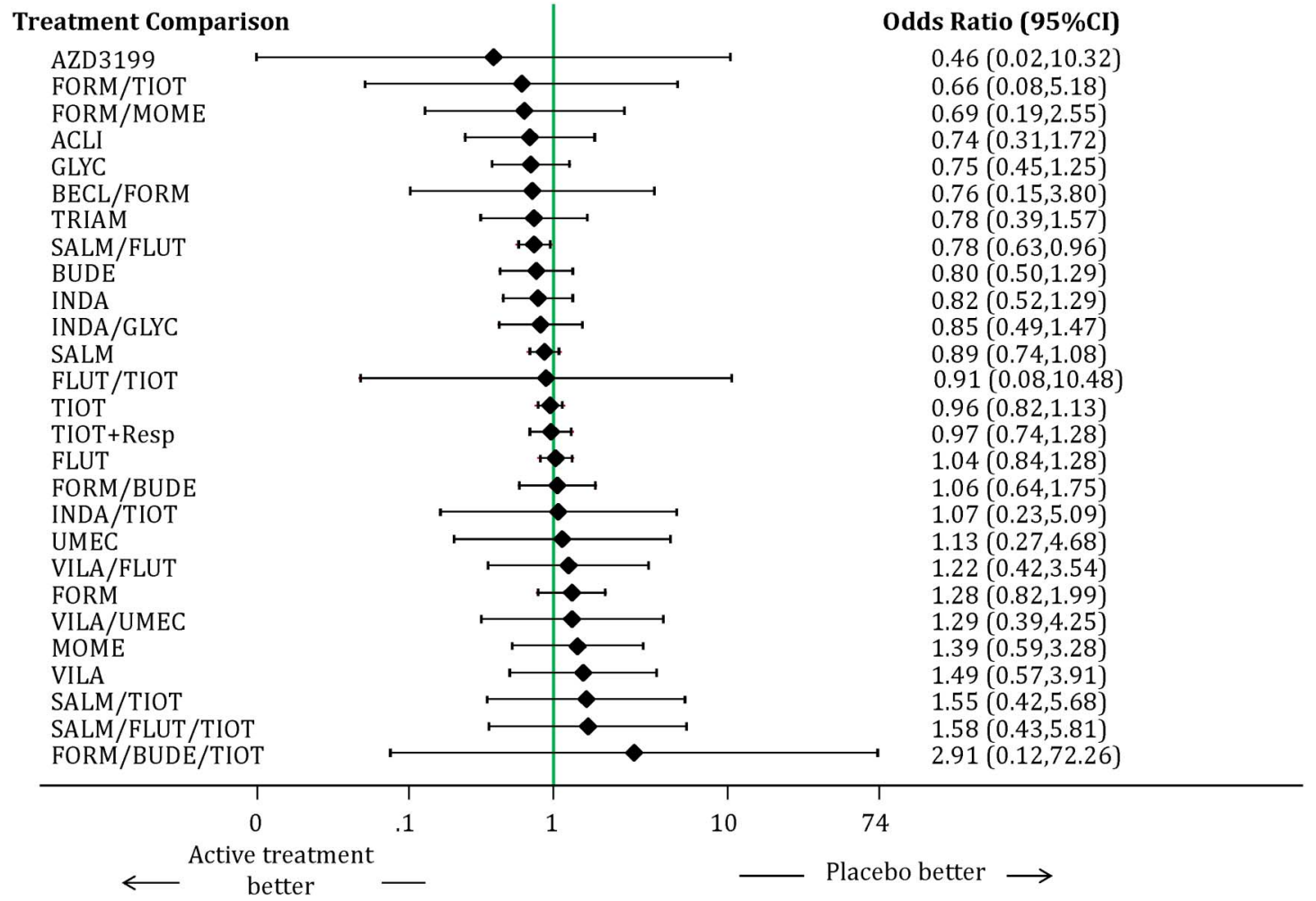

Figure 5 Mortality network meta-analysis forest plot for treatments compared to placebo. The black horizontal lines represent the 95\% Cls of the summary treatment effects AZD3199, AZD3199 (ultra LABA); FORM/TIOT, formoterol/ tiotropium; FORM/ MOME, formoterol/mometasone; ACLI, aclidinium bromide; GLYC, glycopyrronium bromide; BECL/FORM, beclomethasone/ formoterol; TRIAM, triamcinolone acetonide; SALM/FLUT, salmeterol/fluticasone; BUDE, budesonide; INDA, indacaterol; INDA/ GLYC, indacaterol/glycopyrronium; SALM, salmeterol; FLUT/TIOT, fluticasone/tiotropium; TIOT, tiotropium; TIOT+Resp, Tiotropium Respimat (Soft Mist Inhaler); FLUT, fluticasone; FORM/BUDE, formoterol/budesonide; INDA/TIOT, indacaterol/ tiotropium; UMEC, umeclidinium; VILA/FLUT, vilanterol/fluticasone; FORM, formoterol; VILA/UMEC, vilanterol/umeclidinium; MOME, mometasone; VILA, vilanterol; SALM/TIOT, salmeterol/tiotropium; SALM/FLUT/TIOT, salmeterol/fluticasone/tiotropium; FORM/BUDE/TIOT, formoterol/budesonide/tiotropium.

a low risk of bias on the randomisation and allocation concealment components. For this network meta-analysis, fluticasone, indacaterol/glycopyrronium and mometasone/formoterol were the most effective agents at reducing the risk of moderate-to-severe COPD exacerbations.

Our results are similar to a previously published network meta-analysis funded by industry (Merck, Dhome and Nycomed) that included 35 RCTs with 26786 patients and concluded that combination therapy with an ICS and LABA is likely superior to single therapy regarding exacerbations. ${ }^{9}$ Inclusion criteria included patients with moderate-to-severe COPD and trials of at least 24 weeks' duration. A second network meta-analysis of inhaled drugs for COPD in trials of at least 4 weeks' duration concluded that ICS/LABA combination therapy reduced exacerbations only in patients with low forced expiratory volume. ${ }^{8}$ Differences in study eligibility will lead to slightly different network meta-analysis results, reliability and applicability, due to variations in the network of trials.

We also analysed all-cause mortality in a network meta-analysis and found that the most effective agent was fluticasone/salmeterol because it had a decreased risk of mortality compared with the other agents. These results were consistent when we limited the analysis to those studies with a low risk of bias. We also conducted a network meta-analysis on cardiovascular-related mortality and found that use of tiotropium Handihaler and/or tiotropium Soft Mist Inhalers increased the risk compared with some of the other agents. However, in our sensitivity analysis including only studies with a low risk of allocation concealment or randomisation bias no statistically significant results were observed, suggesting that these particular results should be interpreted with caution.

Our mortality results are different, yet the cardiovascular-related mortality results are similar to a previously published network meta-analysis including 42 trials (52 516 patients) of at least 24 weeks' duration involving patients allocated to tiotropium Soft Mist Inhalers, tiotropium Handihaler, ICS+LABA, LABA, ICS or placebo. ${ }^{47}$ Patients receiving the tiotropium Soft Mist Inhalers had the greatest risk of mortality overall, as well as cardiovascular-related mortality.

A recent Cochrane review and network meta-analysis compared four classes of long-acting inhalers for COPD 
(ICS, LABA, ICS/LABA combination and LAMA) for two efficacy outcomes: mean trough forced expiratory volume in $1 \mathrm{~s}\left(\mathrm{FEV}_{1}\right)$ and mean total score on the St George's Respiratory Questionnaire (SGRQ) in trials of at least 24 weeks' duration. ${ }^{48}$ In their review, 71 RCTs with 73062 patients were included. As this recent Cochrane review and network meta-analysis did not examine outcomes pertaining to either exacerbations or mortality, there is no overlap in results with our review.

We found that the following were the most harmful agents for being associated with increasing risk of pneumonia: fluticasone/salmeterol, fluticasone/vilanterol and fluticasone according to the SUCRA. These results are consistent with a recent Cochrane review on ICS, LABA and ICS/LABA combination which looked at pneumonia in patients with COPD in trials of at least 12 weeks' duration. ${ }^{49}$ Those study authors found an increased risk of pneumonia with fluticasone use versus placebo and for any fluticasone/LABA combination versus LABA alone. However, our pneumonia sensitivity analysis including studies with a low risk of bias found that the most harmful agents that increased the risk of pneumonia were budesonide/formoterol, beclomethasone/formoterol, and fluticasone/salmeterol. Of note, we included 132 more studies comprising 56727 more patients than the previous Cochrane reviews (see online supplementary appendix 14).

We found no statistically significant differences in risks of serious arrhythmia across any of the compared agents in our rapid review. This finding is clinically important as clinicians have raised concerns about increasing risk of arrhythmia with use of LABA. ${ }^{50-52}$ We are unaware of any other network meta-analysis that examines this outcome for patients with COPD. We also attempted a network meta-analysis for the dyspnoea outcome, yet the results were unreliable so are not presented here, despite numerous subgroup and sensitivity analyses. Such an analysis should be attempted in the future, perhaps utilising advanced individual patient data network meta-analysis techniques that are currently emerging.

There are some limitations of our systematic review that are worth noting. First, we are aware of 21 new trials that have been published in 16 papers since our original literature search in December of 2013 (see online supplementary appendix 15). This is particularly apparent for the LABA/LAMA combinations. The number of new trials that would be included for each outcome are: 4 trials with 157 patients experiencing moderate-to-severe exacerbations (comparisons include LAMA vs LAMA, ICS/LABA vs LABA, and LABA/LAMA vs LAMA vs LABA vs placebo); 16 trials with 104 patients for mortality (comparisons include LABA vs LABA vs placebo, ICS/LABA vs LABA, ICS/LABA vs LAMA, ICS/LABA vs ICS/LABA, and LABA/LAMA vs LAMA vs LABA vs placebo); 16 trials with 148 patients experiencing pneumonia (comparisons include LABA vs LABA vs placebo, ICS/LABA vs LABA, ICS/LABA vs LAMA, ICS/LABA vs ICS/LABA, and LABA/LAMA vs LAMA vs LABA vs placebo); 13 trials with 125 patients experiencing serious arrhythmia (comparisons include LAMA vs LAMA, ICS/ LABA vs LABA, ICS/LABA vs ICS/LABA, and LABA/ LAMA vs LAMA vs LABA vs placebo); and 7 trials with 11 patients experiencing cardiovascular-related mortality (comparisons include ICS/LABA vs ICS/LABA, and LABA/LAMA vs LAMA vs LABA vs placebo). However, the current review is one of the largest published network meta-analyses ${ }^{53}$ and we have included 208 RCTs and 134692 patients and we believe that our results for the other agents are valid. Second, many of the included RCTs were at a high risk of bias for many of the Cochrane risk-of-bias criteria, especially for important items such as random sequence generation and allocation concealment, which are imperative for the internal validity of a RCT. In order to address this limitation, we conducted a sensitivity analysis for all outcomes, which focused on inclusion of studies with a low risk of bias in the analysis. Third, we were unable to explore other important effect modifiers, such as duration of treatment administration, as this was inconsistently reported across the included RCTs. Fourth, given the inconsistency across the data, we could not complete a network meta-analysis for risk of moderate-to-severe exacerbations overall. Fifth, we limited inclusion to RCTs published in English, yet this has not been shown to bias meta-analysis results in the past. ${ }^{13}$ Finally, we were unable to calculate the PrI for all outcomes, due to the small number of studies included in the exacerbations and sensitivity analyses.

In conclusion, tiotropium/budesonide/formoterol inhaled combination therapy reduces risk of moderate-to-severe exacerbations in patients having already experienced a COPD-related exacerbation in the past year. Inhaled fluticasone/salmeterol reduces risk of mortality, yet may increase risk of pneumonia. Tiotropium may increase risk of cardiovascular-related mortality. These agents likely do not increase risk of serious arrhythmia. Future research should update our review to include studies examining the LABA/LAMA combination, as well as the dyspnoea outcome, as we were presently unable to conduct a network meta-analysis on these.

\section{Author affiliations}

${ }^{1}$ Knowledge Translation Program, Li Ka Shing Knowledge Institute,

St. Michael's Hospital, Toronto, Ontario, Canada

${ }^{2}$ Epidemiology Division, Dalla Lana School of Public Health, University of Toronto, Toronto, Ontario, Canada

${ }^{3}$ Ottawa Hospital Research Institute, Center for Practice Changing Research Building, The Ottawa Hospital-General Campus, Ottawa, Ontario, Canada

${ }^{4}$ Alberta Health Services, Edmonton, Alberta, Canada

${ }^{5}$ Department of Geriatric Medicine, University of Toronto, 27 Kings College Circle, Toronto, Ontario, Canada

Acknowledgements The authors thank Becky Skidmore for conducting the literature search and Heather McDonald for peer reviewing the search, Inthuja Selvaratnam for formatting the paper, and Alissa Epworth for obtaining the full-text articles. They also thank Sandra Knowles for all of her support and useful feedback on the draft manuscript. 
Contributors ACT conceived and designed the study, helped obtain funding for the study, screened citations, abstracted data, guided the analysis, interpreted the results and drafted the manuscript. LS coordinated the review, screened citations and full-text articles, abstracted data, cleaned the data, wrote sections of the manuscript, and edited the manuscript. A-AV analysed and interpreted the data, wrote sections of the manuscript and edited the manuscript. FY screened citations and full-text articles, abstracted data, appraised quality, cleaned the data, and edited the manuscript. PAK abstracted data, appraised quality, cleaned the data and edited the manuscript. AS screened citations and full-text articles, abstracted data, helped clean the data and edited the manuscript. CN screened full-text articles, abstracted data and edited the manuscript. JA screened citations and full-text articles, abstracted data and edited the manuscript. KM abstracted data and edited the manuscript. JD screened citations and full-text articles, and edited the manuscript. RC abstracted data, helped clean the data and edited the manuscript. SES conceived and designed the study, helped obtain funding for the study, guided the analysis, interpreted the results, and edited the manuscript. All authors read and approved the final paper.

Funding This study was funded by the Ontario Ministry of Health and Long-Term Care Health System Research Fund. ACT is funded by a Canadian Institutes of Health Research (CIHR)/Drug Safety and Effectiveness Network New Investigator Award in Knowledge Synthesis. SES is funded by a Tier 1 Canada Research Chair in Knowledge Translation. A-AV is funded by the CIHR Banting Postdoctoral Fellowship Program.

Competing interests None declared.

Provenance and peer review Not commissioned; externally peer reviewed.

Data sharing statement The full data set is available, including data from the 20 included unpublished studies, on request from the corresponding author SES, sharon.straus@utoronto.ca.

Open Access This is an Open Access article distributed in accordance with the Creative Commons Attribution Non Commercial (CC BY-NC 4.0) license, which permits others to distribute, remix, adapt, build upon this work noncommercially, and license their derivative works on different terms, provided the original work is properly cited and the use is non-commercial. See: http:// creativecommons.org/licenses/by-nc/4.0/

\section{REFERENCES}

1. Welsh EJ, Cates CJ, Poole P. Combination inhaled steroid and long-acting beta2-agonist versus tiotropium for chronic obstructive pulmonary disease. Cochrane Database Syst Rev 2013;5:CD007891.

2. Karner C, Cates CJ. Combination inhaled steroid and long-acting beta(2)-agonist in addition to tiotropium versus tiotropium or combination alone for chronic obstructive pulmonary disease. Cochrane Database Syst Rev 2011;(3):CD008532.

3. Nannini LJ, Cates CJ, Lasserson TJ, et al. Combined corticosteroid and long-acting beta-agonist in one inhaler versus long-acting beta-agonists for chronic obstructive pulmonary disease. Cochrane Database Syst Rev 2007;(4):CD006829.

4. Nannini LJ, Poole P, Milan SJ, et al. Combined corticosteroid and long-acting beta(2)-agonist in one inhaler versus inhaled corticosteroids alone for chronic obstructive pulmonary disease. Cochrane Database Syst Rev 2013;8:CD006826.

5. Nannini LJ, Poole P, Milan SJ, et al. Combined corticosteroid and long-acting beta-agonist in one inhaler versus placebo for chronic obstructive pulmonary disease. Cochrane Database Syst Rev 2013;11:CD003794.

6. Cope S, Donohue JF, Jansen JP, et al. Comparative efficacy of long-acting bronchodilators for COPD: a network meta-analysis. Respir Res 2013;14:100.

7. Karabis A, Lindner L, Mocarski M, et al. Comparative efficacy of aclidinium versus glycopyrronium and tiotropium, as maintenance treatment of moderate to severe COPD patients: a systematic review and network meta-analysis. Int $J$ Chron Obstruct Pulmon Dis 2013:8:405-23.

8. Puhan MA, Bachmann LM, Kleijnen J, et al. Inhaled drugs to reduce exacerbations in patients with chronic obstructive pulmonary disease: a network meta-analysis. BMC Med 2009;7:2

9. Mills EJ, Druyts E, Ghement I, et al. Pharmacotherapies for chronic obstructive pulmonary disease: a multiple treatment comparison meta-analysis. Clin Epidemiol 2011;3:107-29.
10. Shamseer L, Moher D, Clarke M, et al. Preferred reporting items for systematic review and meta-analysis protocols (PRISMA-P) 2015: elaboration and explanation. BMJ 2015;349:g7647.

11. Ontario Drug Policy Research Network. Drug Class Review: inhaled corticosteroids (ICS) + long-acting beta-agonists (LABA) combination products for treatment of chronic obstructive pulmonary disease (COPD). http://www.odprn.ca/wp-content/uploads/2014/01/ ODPRN-SR-protocol-final-2.pdf (accessed Jan 2015).

12. Khan S, Mascarenhas A, Dufresne K, et al. ODPRN. Final report: inhaled corticosteroids and long acting beta agonists (ICS/LABA) for the treatment of chronic obstructive pulmonary disease (COPD). http://www.odprn.ca/wp-content/uploads/2015/01/ICS-LABA-COPDQualitative-Report-Final-16_01_15.pdf (accessed Jan 2015).

13. Morrison A, Polisena J, Husereau D, et al. The effect of English-language restriction on systematic review-based meta-analyses: a systematic review of empirical studies. Int $J$ Technol Assess Health Care 2012;28:138-44.

14. Hopewell S, Clarke MJ, Stewart L, et al. Time to publication for results of clinical trials. The Cochrane Library. 2007.

15. Sampson M, McGowan J, Cogo E, et al. An evidence-based practice guideline for the peer review of electronic search strategies. $J$ Clin Epidemiol 2009;62:944-52.

16. Kew KM, Li T. Long-acting inhaled therapy (beta-agonists, anticholinergics and steroids) for COPD: an overview and network meta-analysis. Cochrane Database Syst Rev 2013;(10): CD010178.

17. Higgins JP, Altman DG, Gotzsche PC, et al. The Cochrane Collaboration's tool for assessing risk of bias in randomised trials. BMJ 2011;343:d5928.

18. Raudenbush SW. Analyzing effect sizes: random effects models In: Cooper LVH, Valentine JC, eds. The handbook of research synthesis and meta-analysis. 2nd edn. New York: Russell Sage Foundation, 2009:295-315

19. Higgins JP, Thompson SG. Quantifying heterogeneity in a meta-analysis. Stat Med 2002;21:1539-58.

20. Higgins JPT, Jackson D, Barrett JK, et al. Consistency and inconsistency in network meta-analysis: concepts and models for multi-arm studies. Res Synth Methods 2012;3:98-110.

21. Veroniki AA, Vasiliadis HS, Higgins JP, et al. Evaluation of inconsistency in networks of interventions. Int $J$ Epidemiol 2013;42:332-45.

22. Song F, Altman DG, Glenny AM, et al. Validity of indirect comparison for estimating efficacy of competing interventions: empirical evidence from published meta-analyses. BMJ 2003;326:472.

23. Bucher HC, Guyatt GH, Griffith LE, et al. The results of direct and indirect treatment comparisons in meta-analysis of randomized controlled trials. J Clin Epidemiol 1997;50:683-91.

24. Riley RD, Higgins JPT, Deeks JJ. Interpretation of random effects meta-analyses. BMJ 2011;342:d549.

25. Higgins JP, Thompson SG, Spiegelhalter DJ. A re-evaluation of random-effects meta-analysis. J $R$ Stat Soc Ser A Stat Soc 2009;172:137-59.

26. Chaimani A, Higgins JP, Mavridis D, et al. Graphical tools for network meta-analysis in STATA. PLoS ONE 2013;8:e76654.

27. Salanti G, Ades AE, loannidis JP. Graphical methods and numerical summaries for presenting results from multiple-treatment meta-analysis: an overview and tutorial. J Clin Epidemio 2011;64:163-71.

28. R Core Team. R: A language and environment for statistica computing. http://www.R-project.org (accessed Jan 2015).

29. Schwarzer G. meta: Meta-Analysis with $R$. R package version 4.0-3 http://CRAN.R-project.org/package=meta (accessed Jan 2015).

30. White IR. Multivariate random-effects meta-regression: updates to mvmeta. Stata J 2011;11:255-70.

31. StataCorp. Stata Statistical Software: Release 13. College Station, TX: StataCorp LP, 2013.

32. Thomas N. Overview: OpenBUGS website. http://www.openbugs net/w/Overview (accessed Jan 2015).

33. Turner RM, Davey J, Clarke MJ, et al. Predicting the extent of heterogeneity in meta-analysis, using empirical data from the Cochrane Database of Systematic Reviews. Int J Epidemio 2012;41:818-27.

34. Troosters T, Sciurba FC, Decramer M, et al. Tiotropium in patients with moderate COPD naive to maintenance therapy: a randomised placebo-controlled trial. NPJ Prim Care Respir Med 2014;24:14003.

35. Tieu J, Bain E, Middleton P, et al. Interconception care for women with a history of gestational diabetes for improving maternal and infant outcomes. Cochrane Database Syst Rev 2013;6:CD010211.

36. Andrade-Castellanos Carlos A, Colunga-Lozano Luis E, Delgado-Figueroa $\mathrm{N}$, et al. Subcutaneous rapid-acting insulin 
analogues for diabetic ketoacidosis. Cochrane Database Syst Rev 2014;9:CD011281.

37. Shen W, Li Y, Zhang Y, et al. Acupuncture for adults with type 2 diabetes mellitus. Cochrane Database Syst Rev 2013:4:CD010491.

38. Sekiya M, Kawayama T, Fukuchi $\mathrm{Y}$, et al. Safety and efficacy of NVA237 once daily in Japanese patients: the GLOW4 trial. Eur Respir J 2012;40(Suppl 56):P2103.

39. Brown J, Crawford Tineke J, Alsweiler J, et al. Myo-inositol for preventing gestational diabetes. Cochrane Database Syst Rev 2015;4:CD011507.

40. Crawford Tineke J, Brown J, Alsweiler J, et al. Different intensities of glycaemic control for women with gestational diabetes mellitus. Cochrane Database Syst Rev 2015;4:CD011624.

41. Flowers N, Hartley L, Todkill D, et al. Co-enzyme Q10 supplementation for the primary prevention of cardiovascular disease. Cochrane Database Syst Rev 2014;12:CD010405.

42. Decramer M, Anzueto A, Kerwin E, et al. Efficacy and safety of umeclidinium plus vilanterol versus tiotropium, vilanterol, or umeclidinium monotherapies over 24 weeks in patients with chronic obstructive pulmonary disease: results from two multicentre, blinded, randomised controlled trials. Lancet Respir Med 2014;2:472-86.

43. Wadbo M, Lofdahl CG, Larsson $\mathrm{K}$, et al. Effects of formoterol and ipratropium bromide in COPD: a 3-month placebo-controlled study. Eur Respir J 2002;20:1138-46.

44. Hanania NA, Feldman G, Zachgo W, et al. The efficacy and safety of the novel long-acting beta2 agonist vilanterol in patients with COPD: a randomized placebo-controlled trial. Chest 2012;142:119-27.
45. Wielders PL, Ludwig-Sengpiel A, Locantore N, et al. A new class of bronchodilator improves lung function in COPD: a trial with GSK961081. Eur Respir J 2013;42:972-81.

46. Martinez FJ, Boscia J, Feldman G, et al. Fluticasone furoate/ vilanterol (100/25; 200/25 mug) improves lung function in COPD: a randomised trial. Respir Med 2013;107:550-9.

47. Dong $\mathrm{YH}$, Lin HH, Shau WY, et al. Comparative safety of inhaled medications in patients with chronic obstructive pulmonary disease: systematic review and mixed treatment comparison meta-analysis of randomised controlled trials. Thorax 2013;68:48-56.

48. Kew KM, Dias S, Cates CJ. Long-acting inhaled therapy (beta-agonists, anticholinergics and steroids) for COPD: a network meta-analysis. Cochrane Database Syst Rev 2014;3:CD010844.

49. Kew KM, Seniukovich A. Inhaled steroids and risk of pneumonia for chronic obstructive pulmonary disease. Cochrane Database Syst Rev 2014;3:CD010115.

50. Insulander $\mathrm{P}$, Juhlin-Dannfelt $\mathrm{A}$, Freyschuss $\mathrm{U}$, et al. Electrophysiologic effects of salbutamol, a beta2-selective agonist. J Cardiovasc Electrophysiol 2004;15:316-22.

51. Salpeter SR, Ormiston TM, Salpeter EE. Cardiovascular effects of beta-agonists in patients with asthma and COPD: a meta-analysis. Chest 2004;125:2309-21.

52. Cazzola M, Matera MG, Donner CF. Inhaled beta2-adrenoceptor agonists: cardiovascular safety in patients with obstructive lung disease. Drugs 2005;65:1595-610.

53. Nikolakopoulou A, Chaimani A, Veroniki AA, et al. Characteristics of networks of interventions: a description of a database of 186 published networks. PLOS ONE 2014;9:e86754. 
Correction: Comparative safety and effectiveness of longacting inhaled agents for treating chronic obstructive pulmonary disease: a systematic review and network metaanalysis

Tricco AC, Strifler L, Veroniki A, et al. Comparative safety and effectiveness of longacting inhaled agents for treating chronic obstructive pulmonary disease: a systematic review and network meta-analysis. BMJ Open 2015;5:e009183. DOI: 10.1136/ bmjopen-2015-009183.

The previous version of this manuscript contains a data extraction error in Table 3 in the line comparing 'BUDE/FORM vs placebo'. Revising this data extraction error in the sample size resulted in a slight change in the second digits of the MA and NMA estimates; however, the overall conclusions for this comparison remain the same.

The line in Table 3 should appear as:

BUDE/FORM vs Placebo NMA OR 0.61 (0.43 to 0.87) MA OR $0.52(0.35$ to 0.79$) \quad 1$ (510)

Instead of:

BUDE/FORM vs Placebo NMA OR 0.64 (0.45 to 0.91) MA OR 0.55 (0.36 to 0.83) 1 (519)

In addition, the NMA odds ratio and confidence intervals are also plotted in Figure 4 of the paper. A revised version of Figure 4 is attached.

\author{
Treatment Comparison \\ TIOT/BUDE/FORM vs Placebo \\ INDA/GLYC vs Placebo \\ FLUT/VILA vs Placebo \\ TIOT/FLUT/SALM vs Placebo \\ BUDE/FORM vs Placebo \\ TIOT vs Placebo \\ FLUT/SALM vs Placebo \\ BECLO/FORM vs Placebo \\ TIOT/SALM vs Placebo \\ BUDE vs Placebo \\ VILA vs Placebo \\ GLYC vs Placebo \\ INDA vs Placebo \\ SALM vs Placebo \\ FORM vs Placebo \\ FLUT vs Placebo
}

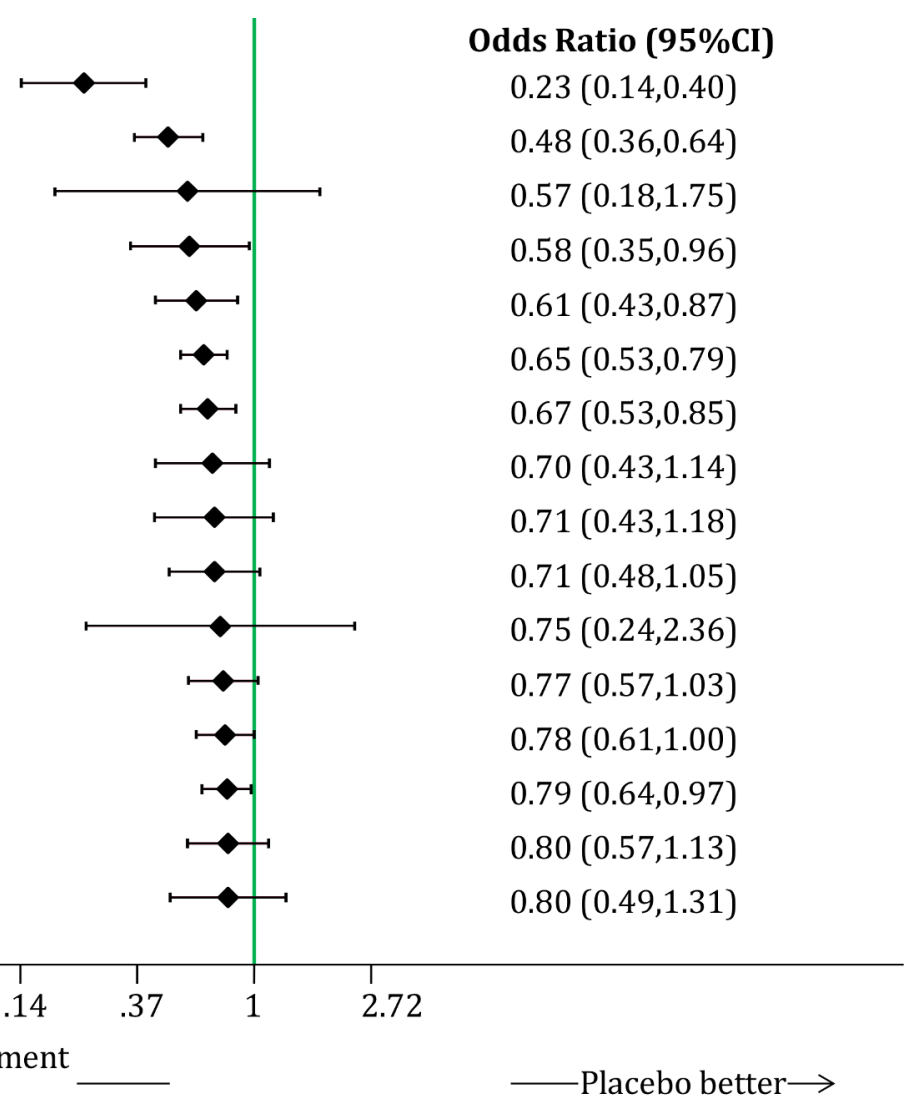

Open access This is an open access article distributed in accordance with the Creative Commons Attribution Non Commercial (CC BY-NC 4.0) license, which permits others to distribute, remix, adapt, build upon this work non-commercially, and license their derivative works on different terms, provided the original work is properly cited, 
appropriate credit is given, any changes made indicated, and the use is non-commercial. See: http://creativecommons.org/ licenses/by-nc/4.0/.

(C) Author(s) (or their employer(s)) 2019. Re-use permitted under CC BY-NC. No commercial re-use. See rights and permissions. Published by BMJ.

BMJ Open 2019;9:e009183corr1. doi:10.1136/bmjopen-2015-009183corr1

A Check for updates 